\title{
A Modified Three-Port Laparoscopic Cholecystectomy: Shifting the Third Port to the Umbilicus
}

\author{
Jianming Zhu*, Jianping Huang \\ Department of General Surgery, Shuguang Hospital Affiliated to Shanghai University of traditional Chinese \\ Medicine, Shanghai, China \\ Email: "zhujianming@hotmail.com
}

Received 25 January 2016; accepted 22 February 2016; published 25 February 2016

Copyright (C) 2016 by authors and Scientific Research Publishing Inc.

This work is licensed under the Creative Commons Attribution International License (CC BY). http://creativecommons.org/licenses/by/4.0/

(c) (i) Open Access

\begin{abstract}
Aim: The aim is to shift the $\mathbf{5} \mathbf{~ m m}$ port from the right upper abdomen to the umbilicus in threeport laparoscopic cholecystectomy to obtain better cosmetic results. Methods: The three ports in conventional three-port laparoscopic cholecystectomy were placed in a $10 \mathrm{~mm}$ epigastric incision, a $5 \mathrm{~mm}$ umbilical incision and a $5 \mathrm{~mm}$ right upper abdominal incision. Our modified method involved movement of the $5 \mathrm{~mm}$ incision at the right upper abdomen to the umbilicus. The clinical data of 67 patients undergoing modified three-port laparoscopic cholecystectomy and 82 patients undergoing conventional three-port laparoscopic cholecystectomy in our hospital between February 2013 and April 2015 were collected, including operating time, intra-operative blood loss, need for conversion to open cholecystectomy, postoperative wound infection, length of hospital stay and satisfaction with cosmetic outcome. Results: One patient in the modified group and two in the conventional group were converted to open surgery due to celiac adhesion. There were no significant differences regarding operating time and blood loss between the two groups. Postoperative stay and wound infection were similar in the two groups, however, patients in the modified group were more satisfied with their cosmetic outcome $(P=0.0006)$. Conclusion: Movement of the $5 \mathrm{~mm}$ port from the right upper abdomen to the umbilicus in conventional laparoscopic cholecystectomy can be performed with acceptable operative outcomes and superior cosmetic results.
\end{abstract}

\section{Keywords}

Laparoscopic Cholecystectomy, Port, Modification

${ }^{*}$ Corresponding author. 


\section{Introduction}

Laparoscopic cholecystectomy is the gold standard for the surgical removal of the gallbladder as it results in a shorter hospital stay, lower levels of postoperative pain, a speedy return to work, superior cosmetic results and significantly lower morbidity rates [1]-[3]. In 1985, Prof. Dr. Erich Mühe of Germany performed the first laparoscopic cholecystectomy (LC). The general indications for laparoscopic cholecystectomy are the same as those for the corresponding open procedure. Although laparoscopic cholecystectomy is originally reserved for young and thin patients, it now is also offered to elderly and obese patients; in fact, these latter patients may benefit even more from surgery through small incisions. Initially, the four-port technique is used, but increasingly this has been replaced by techniques using fewer incisions which are less traumatic and have better cosmetic results [4].

Given the reliability and safety of this surgical procedure, conventional three-port laparoscopic cholecystectomy is still the primary option in our hospital. Using the conventional method, the right upper port incision is $5 \mathrm{~mm}$ in length. If this $5 \mathrm{~mm}$ incision is moved to the umbilicus, the cosmetic outcome will be improved as the umbilicus can cover port incisions. We postulate that this modified surgical procedure will be similar to the double incision laparoscopic cholecystectomy, but will not result in instrument collision, gas leak and a larger umbilical incision.

\section{Methods}

\subsection{Patients}

In total, 149 patients underwent laparoscopic cholecystectomy in our Department of Surgery between February 2013 and April 2015. All patients were diagnosed following preoperative abdominal ultrasonography or computerized tomography and underwent the same preoperative processes. All surgical procedures were carried out by a single surgeon who was experienced in laparoscopic surgery. Patients were consented to participate in the research process. This study was approved by the ethics committee of Shuguang hospital affiliated to Shanghai University of Traditional Chinese Medicine (NO.SGH-13075).

Preoperative preparation included a complete history and physical examination, routine laboratory tests and magnetic resonance cholangiopancreatography. Patients with prior upper abdominal surgery, suspected choledocholithiasis, pregnancy, ongoing peritoneal dialysis, pancreatitis, or Mirizzi's syndrome were excluded from laparoscopic surgery. A history of low abdominal surgery, such as appendectomy, hernia repair, anorectal surgery, uterine or adnexal surgery, was not a criterion for exclusion.

The operating time, intra-operative blood loss, need for conversion to open cholecystectomy, postoperative wound infection, length of hospital stay and satisfaction with cosmetic outcome were recorded. Cosmetic outcome was assessed by patients using a numeric visual analog scale from 1 to 5 (worst to best) on the day of discharge.

\subsection{Operative Technique}

All procedures were performed under general anesthesia and patients were placed in the standard position for laparoscopic cholecystectomy. After performing two $5 \mathrm{~mm}$ infra-umbilical incisions at 8 o'clock and 2 o'clock, respectively, a $\mathrm{CO}_{2}$ pneumoperitoneum was created through the 8 o'clock incision with a Veress needle, and a 5 $\mathrm{mm}$ port was then placed in the umbilicus (Figure 1, Figure 2). The intra-abdominal pressure was maintained at 11 - $14 \mathrm{mmHg}$ and a $5 \mathrm{~mm}$ laparoscope was inserted in this port to guide placement of the 10 mm epigastric port. Another $5 \mathrm{~mm}$ port was then placed through the 2 o'clock incision and a standard cholecystectomy was performed (Figure 3). The specimen was removed via the $10 \mathrm{~mm}$ epigastric incision which was then sutured with polypropylene.

The difference between the conventional laparoscopic cholecystectomy and the modified laparoscopic cholecystectomy was that one of the $5 \mathrm{~mm}$ ports was located at the right hypochondrium anterior to the axillary line and $3 \mathrm{~cm}$ below the costal margin.

\subsection{Statistical Analysis}

All statistical analyses were performed using IBM SPSS statics 19.0. Ordinal variables were calculated as me- 




Figure 1. Ports position.

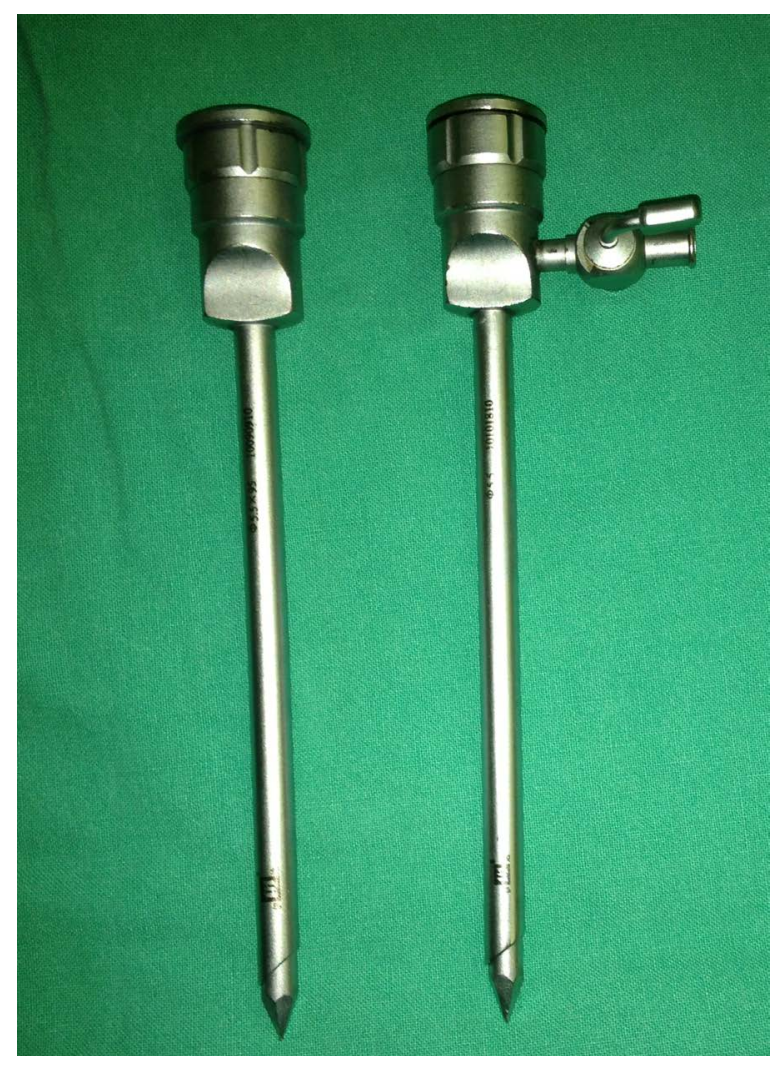

Figure 2. Umbilical $5 \mathrm{~mm}$ ports. 


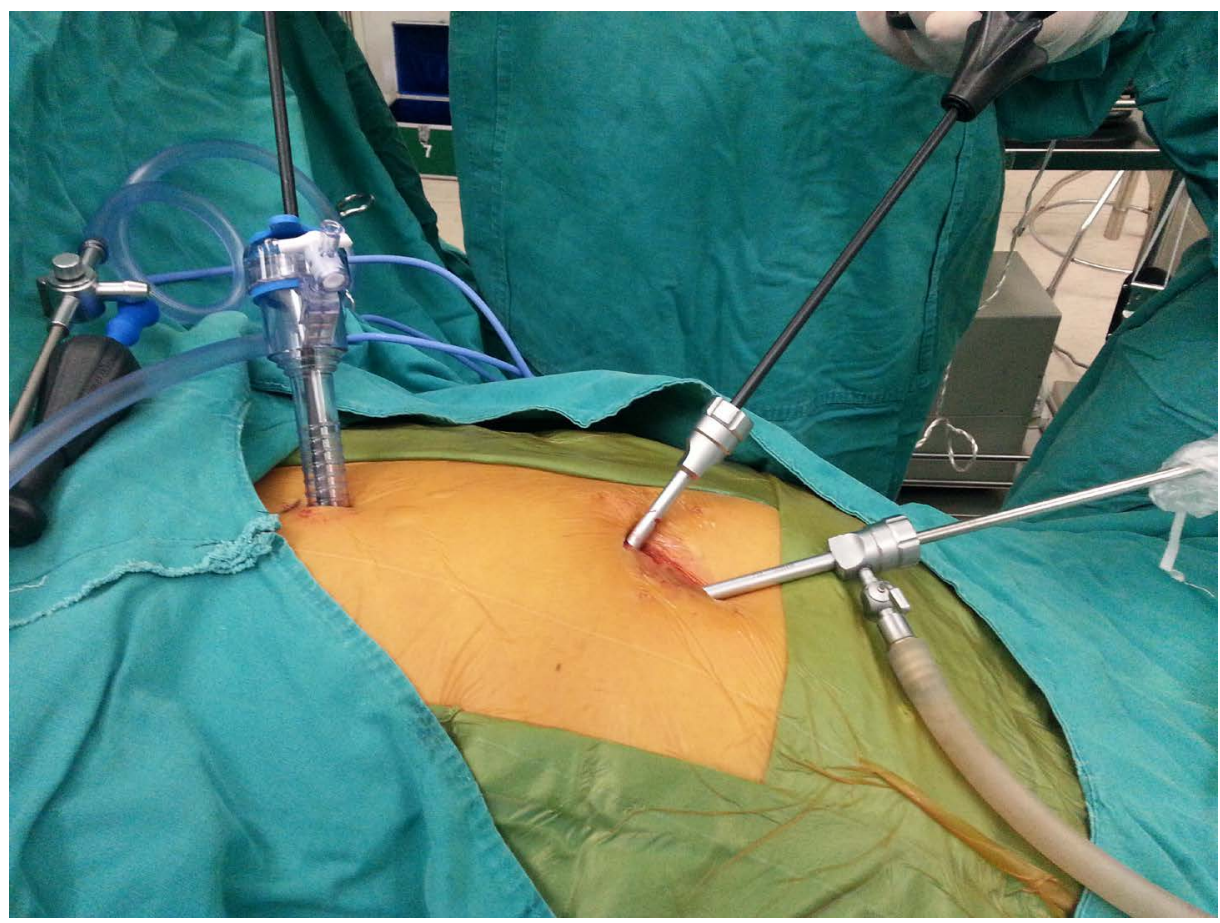

Figure 3. Performing the chelecystectomy.

dian (range) and compared using the Student's $t$ test. This test was also used to analyze differences in operating time, blood loss, hospital stay and cosmetic outcomes between the two groups. The Chi-square test was used to evaluate the effects of gender, disease history, conversion to open surgery and wound infection. A value of $P<$ 0.05 was considered statistically significant.

\section{Results}

There were 98 female and 51 male patients. The age range was 24 - 71 years, with a mean age of 48.52 years. Conventional three-port laparoscopic cholecystectomy was performed in 82 patients, while modified three-port laparoscopic cholecystectomy was performed in 67 patients. Twenty-five patients were diagnosed with acute cholecystitis and 124 patients were diagnosed with chronic cholecystitis. Five patients in the conventional group and 3 in the modified group underwent low abdominal surgery (Table 1).

Complications such as bile duct damage and massive bleeding were not observed. Two patients in the conventional group and one patient in the modified group were converted to open surgery due to celiac adhesion. There were no apparent differences in blood loss between the two groups. The modified three-port procedure was completed in a similar time to the conventional three-port method.

Four patients developed epigastric wound inflammation which was treated with conservative therapy. No significant differences in wound infection and length of hospital stay were observed between the two groups. Patients in the modified group scored higher than those in the conventional group in terms of cosmetic satisfaction $(P<0.05)$ (Table 2).

\section{Discussion}

Although laparoscopic cholecystectomy is initially performed with four incisions, over time there has been a tendency to reduce the number of incisions in order to achieve better cosmetic results [2] [4].

The primary advantage of a single incision laparoscopic cholecystectomy (SILC) is cosmesis. However, SILC is still a relatively new technique and is more difficult than standard laparoscopic cholecystectomy [3] [4]. Long-term outcomes of SILC have yet to be determined. One of these long-term outcomes is port-site incisional hernia which typically occurs as a late postoperative complication [5] [6]. Laparoscopic surgeons agree that the diameter of the cannula or port is related to the development of port-site incisional hernia [7]-[9]. 
Table 1. Patient characteristics.

\begin{tabular}{cccc}
\hline & Conventional (n= 82) & Altered (n= 67) & p \\
\hline Gender & & & \\
Male (n) & 34 & 17 & 0.0558 \\
Female (n) & 48 & 50 & \\
Mean age (year) & $50.23 \pm 10.88$ & $46.43 \pm 12.61$ & 0.0503 \\
Disease history & & & \\
Acute (n) & 18 & 7 & 0.0784 \\
Chronic (n) & 64 & 60 & \\
Low abdominal operation (n) & 5 & 3 & 0.7306 \\
\hline
\end{tabular}

Table 2. Operative and post-operative data.

\begin{tabular}{cccc}
\hline & Conventional (82) & Altered (67) & p \\
\hline Operating time (min) & $47.10 \pm 13.71$ & $51.21 \pm 13.86$ & 0.0720 \\
Blood loss (ml) & $16.12 \pm 16.87(0-60)$ & $18.19 \pm 17.17(0-50)$ & 0.4606 \\
Conversion to open (n) & 2 & 1 & 1.0000 \\
Wound infection (n) & 2 & 2 & 1.0000 \\
Hospital stay (d) & $3.55 \pm 0.92(2-5)$ & $3.81 \pm 0.68(2-5)$ & 0.0587 \\
Cosmetic outcome & $3.26 \pm 0.80(1-5)$ & $3.73 \pm 0.86(2-5)$ & 0.0006 \\
\hline
\end{tabular}

*Statistical significance

Natural orifice trans-luminal surgery is introduced by the authors due to its superior cosmetic outcome and technical feasibility [2] [10]. However, this technique is still controversial as it requires a multidisciplinary team, a long and difficult surgical procedure and there are ethical problems related to the trans-vaginal route [2].

Double incision laparoscopic cholecystectomy (DILC) performed by an inexperienced surgeon is as successful and safe as traditional laparoscopic cholecystectomy [4] [11]. However, two fascial defects converted to a single larger fascial defect more than $10 \mathrm{~mm}$ has the risk of hernia development, although port-site hernia in the umbilicus is not frequently encountered in the clinic [8] [9]. The learning curve for DILC is short, however, one umbilical incision with two ports can easily result in instrument collision [4], and a potential air leak between the ports.

Studies have shown that the three-port technique does not change the rate of conversion or increases the operating time when compared to the four-port technique [12]. With the aim of achieving better cosmetic results, we modify the conventional three-port technique. Although there is no reduction in port number, movement of the 5 $\mathrm{mm}$ port from the right upper abdomen to the umbilicus resulted in scars being hidden in the umbilicus and a larger incision is avoided. In addition, two separate $5 \mathrm{~mm}$ port incisions avoid potential air leaks and do not reduce the solidity of the abdominal wall even without sutures. No increased surgical difficulty or postoperative complications are found.

The umbilical ports are located at the 2 o'clock and 8 o'clock position, respectively, which may have reduced instrument collision. Both $5 \mathrm{~mm}$ ports are not for single use and have compressed ends. The port at 2 o'clock has no gas inflow part, which further shrinks its end and reduces port collision out of the abdomen (Figure 2).

The specimen is removed via the $10 \mathrm{~mm}$ epigastric incision. Generally, the incision is extended to allow easy removal of the specimen, but a larger incision may contribute to a defect in the abdominal wall if not sufficiently sutured. We do not make a $10 \mathrm{~mm}$ incision in the umbilicus as the upper abdominal wall endures less intra-abdominal pressure than the lower abdominal wall in the standing position [13]. Removing the specimen via the epigastric incision is better than via the umbilical incision with regard to the risk of port site hernia.

When compared with conventional three-port laparoscopic cholecystectomy, a modification of the $5 \mathrm{~mm}$ port from the right upper abdomen to the umbilicus did not increase surgical difficulty, postoperative wound infection and hospital stay were similar, and the modification resulted in better cosmetic results. It might also potentially reduce port-site incisional hernia in the umbilicus as compared to SILC or DILC, although further evidence-based research was required.

In conclusion, movement of the $5 \mathrm{~mm}$ port from the right upper abdomen to the umbilicus in conventional la- 
paroscopic cholecystectomy can be performed with acceptable operative outcomes and superior cosmetic results.

\section{Acknowledgements}

We thank doctor Yining Xu for his assisitance in surgical operations. We also thank language editor Jingyun Ma for her significant revision of the manuscript.

\section{Competing Interest}

We have no conflict of interest to declare.

\section{Authors' Contributions}

Jianming Zhu conceived and designed the study, conducted the analyses, data interpretation and manuscript production. Jianping Huang participated in study design, analytic method design and data interpretation, and approved the final submitted manuscript. Surgical operations were performed by Jiangming Zhu under the guidance of Jianping Huang.

\section{Funding}

There was no funding received for this work.

\section{References}

[1] Hao, L., Liu, M., Zhu, H., et al. (2012) Single-Incision versus Conventional Laparoscopic Cholecystectomy in Patients with Uncomplicated Gallbladder Disease: A Meta-Analysis. Surgical Laparoscopy, Endoscopy \& Percutaneous Techniques, 22, 487-497. http://dx.doi.org/10.1097/SLE.0b013e3182685d0a

[2] Pollard, J.S., Fung, A.K. and Ahmed, I. (2012) Are Natural Orifice Transluminal Endoscopic Surgery and Single-Incision Surgery Viable Techniques for Cholecystectomy? Journal of Laparoendoscopic \& Advanced Surgical Techniques, 22, 1-14. http://dx.doi.org/10.1089/lap.2011.0341

[3] Ahmed, M.U., Aftab, A., Seriwala, H.M., et al. (2014) Can Single Incision Laproscopic Cholecystectomy Replace the Traditional Four Port Laproscopic Approach: A Review. Global Journal of Health Science, 6, 119-125. http://dx.doi.org/10.5539/gjhs.v6n6p119

[4] Sabuncuoglu, M.Z., Benzin, M.F., Cakir, T., et al. (2014) Triple, Double- and Single-Incision Laparoscopic Cholecystectomy: A Prospective Study. International Journal of Clinical and Experimental Medicine, 7, 3385-3391.

[5] Marks, J.M., Phillips, M.S., Tacchino, R., et al. (2013) Single-Incision Laparoscopic Cholecystectomy Is Associated with Improved Cosmesis Scoring at the Cost of Significantly Higher Hernia Rates: 1-Year Results of a Prospective Randomized, Multicenter, Single-Blinded Trial of Traditional Multiport Laparoscopic Cholecystectomy vs Single-Incision Laparoscopic Cholecystectomy. Journal of the American College of Surgeons, 216, 1037-1047. http://dx.doi.org/10.1016/j.jamcollsurg.2013.02.024

[6] Alptekin, H., Yilmaz, H., Acar, F., et al. (2012) Incisional Hernia Rate May Increase after Single-Port Cholecystectomy. Journal of Laparoendoscopic \& Advanced Surgical Techniques, 22, 731-737. http://dx.doi.org/10.1089/lap.2012.0129

[7] Zemet, R., Mazeh, H., Grinbaum, R., et al. (2012) Incarcerated Hernia in 11-mm Nonbladed Trocar Site Following Laparoscopic Appendectomy. JSLS, 16, 178-181. http://dx.doi.org/10.4293/108680812X13291597716780

[8] Yamamoto, M., Minikel, L. and Zaritsky, E. (2011) Laparoscopic 5-mm Trocar Site Herniation and Literature Review. JSLS, 15, 122-126. http://dx.doi.org/10.4293/108680811X13022985131697

[9] Bunting, D.M. (2010) Port-site Hernia Following Laparoscopic Cholecystectomy. JSLS, 14, 490-497. http://dx.doi.org/10.4293/108680810X12924466007728

[10] Solomon, D., Shariff, A.H., Silasi, D.A., et al. (2012) Transvaginal Cholecystectomy versus Single-Incision Laparoscopic Cholecystectomy versus Four-Port Laparoscopic Cholecystectomy: A Prospective Cohort Study. Surgical Endoscopy, 26, 2823-2827. http://dx.doi.org/10.1007/s00464-012-2253-0

[11] D’Antonio, D., Franzato, B., Fusco, G., et al. (2013) Double Incision Laparoscopic Cholecystectomy (DILC) with Routinary Intra-Operative Cholangiography (IOC): Less Trauma, Same safety. Report on 30 Consecutive Non-Selected Cases. Updates in Surgery, 65, 109-114. http://dx.doi.org/10.1007/s13304-013-0200-9 
[12] Al-Azawi, D., Houssein, N., Rayis, A.B., et al. (2007) Three-Port versus Four-Port Laparoscopic Cholecystectomy in Acute and Chronic Cholecystitis. BMC Surgery, 7, 8. http://dx.doi.org/10.1186/1471-2482-7-8

[13] Freimann, F.B, Ötvös, J., Chopra, S.S., et al. (2013) Differential Pressure in Shunt Therapy: Investigation of Position-Dependent Intraperitoneal Pressure in a Porcine Model. Journal of Neurosurgery: Pediatrics, 12, 575-581. http://dx.doi.org/10.3171/2013.8.PEDS13205

\section{List of Abbreviations}

SILC: Single incision laparoscopic cholecystectomy

DILC: Double incision laparoscopic cholecystectomy 